\title{
Quistes de cuerda vocal: Experiencia en 44 pacientes del Centro de Voz del Departamento de Otorrinolaringología de la Pontificia Universidad Católica de Chile
}

\author{
Vocal Cord Cysts: Experience in 44 patients from the Voice Center of \\ the Department of Otorhinolaryngology Pontificia Universidad Católica de Chile
}

\author{
Ángela Chuang $\mathrm{Ch}^{1}$, Pedro Badía $\mathrm{V}^{2}$, Carla Napolitano $\mathrm{V}^{2}$.
}

\begin{abstract}
RESUMEN
Introducción: Los quistes de cuerda vocal son una causa relativamente frecuente de disfonía. Su origen es aún controversial, y su diagnóstico y manejo continúan siendo un desafío clínico.

Objetivo: Exponer y analizar las características clínicas de los quistes de cuerda vocal en nuestra serie de pacientes.

Material y método: Estudio retrospectivo descriptivo de los pacientes con diagnóstico de quiste de cuerda vocal atendidos en nuestro centro entre junio de 2012 y diciembre de 2015.

Resultados: Se atendieron 44 pacientes con diagnóstico de quiste de cuerda vocal, lo que representa el $4,32 \%$ de las consultas en nuestro Centro de Voz. La mayoría de los pacientes fueron adultos, y de ellos el 68,29\% correspondió a mujeres. El 34,1\% de los pacientes fueron sometidos a tratamiento quirúrgico con técnica de microfonocirugía. El $75 \%$ de los pacientes operados presentó mejoría en patrón de onda mucosa videolaringoestroboscópica. Todos los pacientes en los que se disponía de encuestas de valoración subjetiva de la voz pre y posoperatorias demostraron mejoría vocal significativa.

Conclusión: Los quistes de cuerda vocal son lesiones que afectan a niños y adultos. La videolaringoestroboscopía es clave en el diagnóstico de estas lesiones, y el tratamiento quirúrgico con microfonocirugía es efectiva en cuanto a resultados vocales desde el punto de vista anatómico y funcional.
\end{abstract}

Palabras clave: Disfonía, quiste de cuerda vocal, fonocirugía, videolaringoestroboscopía.

\begin{abstract}
Introduction: Vocal cord cysts are a relatively frequent cause of dysphonia. Their origin is still controversial, and their diagnosis and management continue to be a clinical challenge.
\end{abstract}

Médico Cirujano, Pontificia Universidad Católica de Chile.

2 Médico del Departamento de Otorrinolaringología, Pontificia Universidad Católica de Chile.

Recibido el 16 de octubre, 2016. Aceptado el 10 de enero, 2017. 
Aim: To describe and analyze the clinical characteristics of vocal cord cysts in our series of patients.

Material and method: Descriptive retrospective study of patients with diagnosis of vocal cord cyst attended in our center between June 2012 and December 2015.

Results: 44 patients had the diagnosis of vocal cord cyst, which represents $4.32 \%$ of the patients that attended our Voice Center during that period. Most of the patients were adults, and among them $68.29 \%$ corresponded to women. $34.1 \%$ of the patients were submitted to surgical treatment with microphonosurgery technique. $75 \%$ of the surgical patients presented an improvement in the pattern of the videolaryngostroboscopic mucosal wave. All the patients in which pre and postsurgical subjective voice assessment polls were available, showed a significant voice improvement.

Conclusion: Vocal cord cysts are lesions that affect both children and adults. The videolaryngostroboscopy evaluation is key in the diagnosis of these lesions, and the surgical treatment with microphonosurgery is effective in terms of anatomical and functional vocal results.

Key words: Dysphonia, vocal cord cyst, phonosurgery, videolaryngostroboscopy.

\section{INTRODUCCIÓN}

Los quistes de cuerda vocal corresponden a lesiones encapsuladas, revestidas de epitelio, ubicadas en la lámina propia de la cuerda vocal. Éstos se clasifican en dos grupos: quistes epidérmicos y quistes de retención mucosos. Los quistes epidérmicos se observan como un aumento de volumen fusiforme de color amarillo o blanco nacarado dentro de la lámina propia (Figura 1). Su origen puede ser congénito 0 adquirido: remanentes celulares de los cuarto y sexto arcos branquiales que quedan en el subepitelio de la cuerda vocal durante el desarrollo embrionario, 0 bien, secundarios a inclusión de tejido epitelial en la mucosa de la cuerda vocal durante el proceso de cicatrización de una lesión fonotraumática. A la histología, los quistes epidérmicos se observan revestidos por epitelio escamoso estratificado $0^{1,2}$.

Por otro lado, los quistes de retención mucosos habitualmente se encuentran en la lámina propia superficial de la cuerda vocal, y se observan como una colección subepitelial bien delimitada, translúcida, de mucus. Se producen por la obstrucción de los conductos de glándulas mucosas, generalmente durante el curso de una infección respiratoria alta 0 asociado a abuso vocal. A la histología se observa que están revestidos por epitelio cilíndrico de tipo respiratorio. Son más frecuentes en adultos, pero también pueden ser diagnosticados en niños.
Los quistes vocales se describen tanto en adultos como en niños, y pueden ser unilaterales o bilaterales. En un estudio de Bouchayer y Cornut ${ }^{3}$ que incluyó a 1.283 pacientes con lesiones laríngeas benignas, la prevalencia de quistes de cuerda vocal fue de $13,6 \%$. La literatura es equívoca al momento de definir si hay

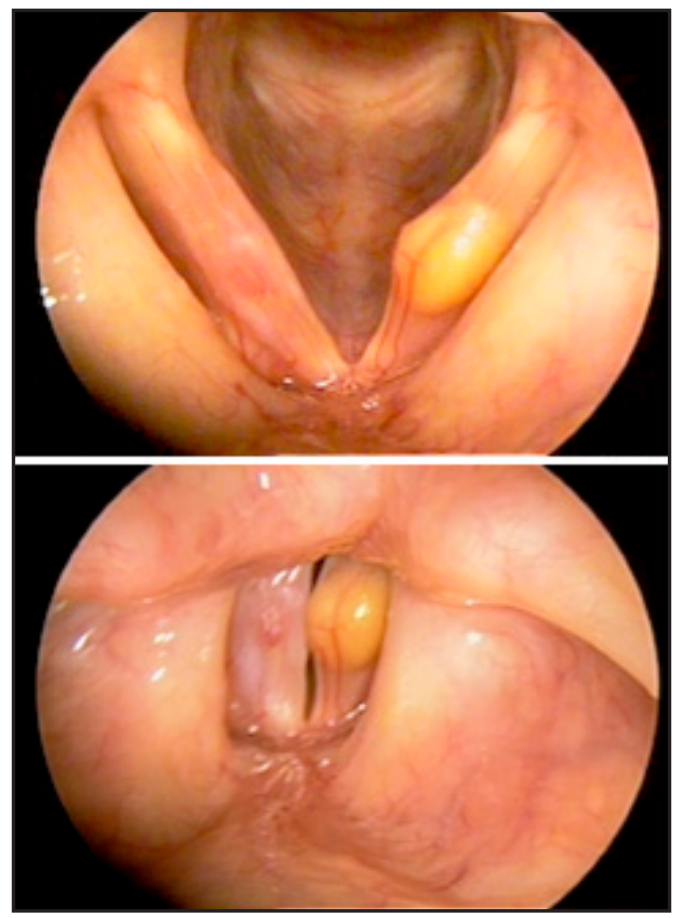

Figura 1. Quiste epidérmico de cuerda vocal izquierda. 
diferencias en su distribución por género, aunque habría una tendencia al predominio en mujeres ${ }^{1,4,5}$.

La presentación clínica más frecuente de los quistes de cuerda vocal es la disfonía. A la videolaringoestroboscopía se observa una lesión generalmente en el tercio medio de la cuerda vocal, asociada a un hiato fonatorio en reloj de arena, y la onda mucosa se encuentra disminuida 0 ausente en relación a la lesión. Puede además existir hiperfunción supraglótica compensatoria. Si el quiste está asociado a una lesión por contragolpe 0 si son quistes bilaterales, pueden confundirse con nódulos vocales. Los quistes no responden al reposo vocal ni al tratamiento fonoaudiológico, requiriendo tratamiento quirúrgico $0^{1,2}$.

\section{OBJETIVO}

Dadas las particularidades de los quistes de cuerda vocal y la insuficiente información respecto a su origen, incidencia y manejo, el objetivo de este estudio es exponer las características clínicas de estas lesiones en nuestra serie de pacientes.

\section{MATERIAL Y MÉTODO}

Se revisaron las fichas clínicas y videolaringoestroboscopías de todos los pacientes con diagnóstico de quiste de cuerda vocal, que fueron atendidos en el Centro de Voz del Departamento de Otorrinolaringología de la Pontificia Universidad Católica de Chile en el período comprendido desde junio de 2012 a diciembre de 2015. Las variables analizadas fueron: género, edad, ocupación, duración de los síntomas vocales, síntomas nasosinusales y gastroesofágicos, reporte de abuso vocal, tabaquismo y alcoholismo, lateralidad de la lesión, lesiones laríngeas asociadas, tratamiento recibido, resultado de biopsia, características videolaringoestroboscópicas pre y posoperatorias, y encuestas de valoración subjetiva de la voz pre y posoperatorias. Se excluyeron aquellos casos en los que el diagnóstico era incierto.

En los pacientes sometidos a videolaringoestroboscopía se utilizó un telelaringoscopio rígido (8 $\mathrm{mm}, 70^{\circ}$ ) acoplado al equipo Laryngeal Strobe model 9400 Pentax medical 9310 HD.
El diagnóstico videolaringoestroboscópico de quiste de cuerda vocal se estableció como un aumento de volumen intracordal uni o bilateral, con bordes bien delimitados, de aspecto translúcido, amarillento o blanco nacarado, que a la estroboscopía demuestra disminución 0 ausencia de la onda mucosa sobre la lesión.

\section{RESULTADOS}

Un total de 1.018 pacientes, 593 mujeres (58,25\%) y 425 hombres (41,75\%) fueronatendidos en el Centro de Voz del Departamento de Otorrinolaringología de la Pontificia Universidad Católica de Chile entre junio de 2012 y diciembre de 2015. De ellos, 44 pacientes presentaron el diagnóstico de quiste de cuerda vocal, correspondiendo al 4,32\% del total. De éstos, $38(86,36 \%)$ fueron estudiados con videolaringoestroboscopía, mientras que los 6 restantes $(13,64 \%)$ fueron estudiados con nasofaringolaringofibroscopía.

Edad y género: De los 44 pacientes con diagnóstico de quiste de cuerda vocal, 41 fueron adultos $(93,18 \%)$ y 3 fueron pediátricos $(6,82 \%)$. De la población adulta, 28 fueron mujeres $(68,29 \%)$ y 13 fueron hombres $(31,71 \%)$. De la población pediátrica, los pacientes se distribuyeron inversamente: 1 niña $(33,3 \%)$ y 2 niños $(66,6 \%)$.

Ocupación: En la Tabla 1 se resumen las principales ocupaciones asociadas a los pacientes con quiste de cuerda vocal. Destacan entre las primeras dos mayorías los estudiantes y los profesores $(38,64 \%)$.

Duración de los síntomas vocales: De todos los pacientes con quiste de cuerda vocal, 40 (90,91\%) consultaron por disfonía, y la duración de sus síntomas se distribuye según la Tabla 2. En los 4 pacientes $(9,09 \%)$ restantes, el diagnóstico fue un hallazgo en la evaluación laringoscópica indicada por otros motivos.

Síntomas nasosinusales y gasestroesofágicos: Fueron reportados síntomas nasosinusales por 25 pacientes $(56,82 \%)$, y síntomas gastroesofágicos por 36 pacientes $(81,82 \%)$. 
Tabla 1. Ocupaciones de pacientes con quiste de cuerda vocal

\begin{tabular}{|lcc|}
\hline & N & $\%$ \\
\hline Estudiante & 10 & 22,7 \\
Profesor & 7 & 15,9 \\
Actividades domésticas & 6 & 13,6 \\
Vendedor & 4 & 9 \\
Ingeniero comercial & 2 & 4,6 \\
Actriz & 1 & 2,3 \\
Otros & 9 & 20,5 \\
Sin registro & 5 & 11,4 \\
\hline Total & 44 & 100 \\
\hline
\end{tabular}

Tabla 2. Duración de síntomas vocales en pacientes con quiste de cuerda vocal

\begin{tabular}{|lrr|}
\hline & N & $\%$ \\
\hline Menor o igual a 12 meses & 29 & 65,9 \\
Entre más de 1 año y 3 años & 7 & 15,9 \\
Más de 3 años & 3 & 6,8 \\
Desde infancia & 3 & 6,8 \\
Sin registro & 2 & 4,6 \\
\hline Total & 44 & 100 \\
\hline
\end{tabular}

Reporte de abuso vocal: El abuso vocal fue reportado por 20 pacientes (45,45\%).

Tabaquismo y alcoholismo: Tabaquismo fue reportado por 20 pacientes $(45,45 \%)$ y alcoholismo por ningún paciente.

Lateralidad de la lesión: Sólo en 1 paciente pediátrico $(2,27 \%)$ se observó presencia bilateral de quistes de cuerdas vocales, los otros 43 pacientes $(97,73 \%)$ presentaron lesión unilateral, siendo en 15 pacientes $(34,09 \%)$ a derecha, en 27 pacientes $(61,36 \%)$ a izquierda, y en 1 paciente $(2,27 \%)$ no se pudo disponer de la lateralidad de la lesión.

Lesiones laríngeas asociadas: En 32 pacientes $(72,7 \%)$ se objetivó la presencia de lesiones laríngeas asociadas (sin considerar quiste de cuerda vocal bilateral), y dentro de este grupo, en 3 pacientes $(9,37 \%)$ se pesquisó más de una lesión asociada (dos pacientes con dos lesiones asociadas, y un paciente con tres lesiones asociadas), contando un total de 36 lesiones asociadas. Entre las más frecuentes se encuentran la lesión por contragolpe, sulcus vocalis y edema de Reinke (Tabla 3).

Tratamiento: A los 44 pacientes que fueron diagnosticados con quiste de cuerda vocal se les indicó cirugía, pero sólo 15 de ellos $(34,09 \%)$ fueron operados finalmente (14 adultos y 1 niño con diagnóstico de quistes bilaterales, operado unilateral). Uno de los pacientes operados (6,67\%) requirió reintervención por recurrencia al año de la primera cirugía.

A todos los pacientes operados se les realizó microfonocirugía con técnica de microflap. Se realizó una cordotomía superficial inmediatamente lateral a la lesión, y luego una disección roma 
Tabla 3. Lesiones laríngeas asociadas

\begin{tabular}{|lrr|}
\hline & N & $\%$ \\
\hline Lesión por contragolpe & 19 & 52,7 \\
Sulcus vocalis contra o bilateral & 5 & 13,8 \\
Edema de Reinke & 3 & 8,3 \\
Leucoplaquia cordal & 2 & 5,6 \\
Vasculodisgenesia & 2 & 5,6 \\
Cicatrices cordales bilaterales & 1 & 2,8 \\
Laringitis crónica & 1 & 2,8 \\
Nódulos vocales & 1 & 2,8 \\
Paresia cordal unilateral & 1 & 2,8 \\
Obs. quiste sacular & 1 & 2,8 \\
\hline Total & 36 & 100 \\
\hline
\end{tabular}

cuidadosa de ella, procurando removerla intacta para evitar la recurrencia. Después de la excisión completa de la lesión se reposicionó el microflap. En los casos en que se produjo la rotura del quiste, se realizó una resección cuidadosa de la cápsula remanente de la lesión. Se observó que los quistes epidérmicos presentaban una consistencia más firme y una cápsula más gruesa que los quistes de retención mucosos, lo que facilitaba su disección, disminuyendo la posibilidad de rotura.

Las lesiones asociadas pesquisadas en la videolaringoestroboscopía fueron confirmadas durante la cirugía, y algunas lesiones asociadas fueron diagnosticadas durante la microlaringoscopía.

Análisis histopatológico: Al analizar la histología de las piezas operatorias, $9(60 \%)$ correspondieron a quistes epidérmicos (descritos como revestidos por epitelio escamoso), 5 (33,3\%) a quistes mucosos (descritos como revestidos por epitelio de tipo respiratorio) y en 1 paciente $(6,67 \%)$ la biopsia no especificó el tipo de quiste (Tabla 4).
Videoestroboscopía preoperatoria: Se dispone de la información de la onda mucosa en 33 de los 38 pacientes $(86,9 \%)$ evaluados con videolaringoestroboscopía. De ellos, 31 pacientes (94\%) mostraron un patrón alterado en la onda mucosa asociada a la lesión. En la Tabla 5 se detallan sus características videolaringoestroboscópicas.

Videoestroboscopía posoperatoria: Los 15 pacientes que fueron sometidos a cirugía fueron evaluados con videolaringoestroboscopía posoperatoria, sin embargo sólo se pudo disponer de la evaluación preoperatoria de 12 de ellos (80\%). Al comparar los hallazgos pre y posquirúrgicos destaca que 9 pacientes (75\%) mostraron cambios favorables: 6 pacientes lograron llegar a una onda mucosa normal, 2 pacientes de onda ausente a disminuida y 1 paciente de onda disminuida a levemente disminuida, y los 3 pacientes restantes (25\%) no mostraron cambios videolaringoestroboscópicos, manteniéndose los 3 con onda mucosa disminuida.

Tabla 4. Tipo de quiste de cuerda vocal

\begin{tabular}{|lcc|}
\hline & N & $\%$ \\
\hline Epidérmico & 9 & 60 \\
Mucoso & 5 & 33,3 \\
No especificado & 1 & 6,7 \\
\hline Total & 15 & 100 \\
\hline
\end{tabular}


Tabla 5. Características de la onda mucosa preoperatoria

\begin{tabular}{|lrr|}
\hline & N & $\%$ \\
\hline Disminuida & 17 & 44,7 \\
Ausente & 13 & 34,2 \\
Normal & 3 & 7,9 \\
Sin dato disponible & 5 & 13,2 \\
\hline Total & 38 & 100 \\
\hline
\end{tabular}

Valoraciones subjetivas de la voz: De los 15 pacientes que fueron sometidos a cirugía, 6 pacientes (40\%) contaban con encuesta de valoración subjetiva de la voz tanto pre como posoperatoria.

- Voice Related Quality of Life (VRQOL) $)^{6}$. El promedio preoperatorio fue de $45 \%$ (DE 17,74) y el promedio posoperatorio fue de $94,17 \%$ (DE 7,17). El 100\% de los pacientes mostró un cambio significativo luego del procedimiento, con un promedio de cambio de $49,17 \%$.

- Voice Handicap Index 10 (VHI-10): El promedio preoperatorio fue de 26,67 puntos (DE 9,34) y el promedio posoperatorio fue de 1,67 puntos (DE $2,21)$. Hubo una disminución significativa en el $100 \%$ de los pacientes en el posoperatorio, con un promedio de cambio de 25 puntos.

- Autopercepción: La autopercepción de la voz fue evaluada con 5 categorías (pobre, suficiente, buena, muy buena, excelente). De los 6 pacientes operados, 5 de ellos ( $83,33 \%$ ) manifestaron en el preoperatorio una "pobre" autopercepción de su voz, con el paciente restante evaluándola como "suficiente". En la evaluación posoperatoria, los 6 pacientes (100\%) calificaron su voz como "buena" o "muy buena".

\section{DISCUSIÓN}

Algunos autores han reportado incidencias de $13 \%-24 \%$ de quistes de cuerdas vocales en pacientes que consultan por disfonía ${ }^{3,8}$, lo que es mayor que lo encontrado en nuestro estudio $(4,32 \%)$, ya que estos autores consideraron sólo pacientes con lesiones benignas de cuerdas vocales, mientras que nuestra serie incluyó a todos los pacientes que fueron atendidos en el Centro de Voz del Departamento de Otorrinolaringología de la
Pontificia Universidad Católica de Chile, tanto con patología benigna fonotraumática como también neoplásica, neurológica y traumática, entre otras.

En nuestra serie, los quistes de cuerda vocal en los adultos fueron más frecuentes en mujeres (68,3\% mujeres versus $31,7 \%$ hombres), mientras que en los pacientes pediátricos, esta proporción se invierte: $33,3 \%$ niñas y $66,6 \%$ niños, fenómeno que está descrito en la literatura ${ }^{4}$. Este mismo comportamiento se observa en los nódulos vocales, cuya etiología se ha asociado al fonotrauma, entre otros factores. La predominancia de los nódulos vocales en el género masculino durante infancia tendría relación con el abuso vocal más frecuente en los varones en la etapa infantil, mientras que en los adultos, la disminución de la frecuencia fundamental de la voz en los hombres después de la pubertad coincide con el predominio de los nódulos vocales en el género femenino. Por lo tanto, si bien el diagnóstico de quiste de cuerda vocal en la población pediátrica puede asociarse a un origen congénito, esta diferencia en incidencia según género apoyaría el hecho que el fonotrauma también se asocia a estas lesiones.

En general se considera que la aparición de lesiones benignas fonotraumáticas de las cuerdas vocales se asocia a la coexistencia de abuso vocal y factores inflamatorios de la mucosa laríngea, como patología respiratoria, gastrointestinal, tabaquismo y abuso del alcohol. En nuestro estudio, el abuso vocal fue reportado por el $45,5 \%$ de los pacientes. La presencia de síntomas nasosinusales fue reportada por el $56,8 \%$ de los pacientes, la presencia de síntomas gastroesofágicos fue referida por el $81,8 \%$ de los pacientes, y el tabaquismo estuvo presente en casi la mitad de los pacientes $(45,45 \%)$. El hecho que la mayoría de nuestros pacientes reporte la presencia de factores inflamatorios de la mucosa laríngea, y la mitad de ellos también reconozca abuso vocal, apo- 
yaría la hipótesis que ambos factores favorecerían la aparición de los quistes de cuerda vocal.

En todos nuestros pacientes sometidos a cirugía se confirmó el diagnóstico de quiste de cuerda vocal, no existiendo ningún caso en que se cambiara el diagnóstico en el intraoperatorio. En este sentido, la videolaringoestroboscopía es útil en el diagnóstico de estas lesiones, especialmente cuando nos enfrentamos a lesiones bilaterales que pueden ser confundidas con nódulos vocales, siendo la reducción de la amplitud 0 ausencia de la onda mucosa una característica determinante en los quistes de cuerda vocal.

Los quistes de cuerda vocal pueden coexistir con otras lesiones laríngeas, como puentes mucosos y sulcus vocalis, entre otros. Martins y $\mathrm{Col}^{4}$ reportaron la presencia de lesiones laríngeas asociadas en el $30 \%$ de los pacientes con quiste de cuerda vocal. En nuestro estudio, en el $72,7 \%$ de los pacientes se objetivó la presencia de lesiones laríngeas asociadas. Si se excluyen las lesiones por contragolpe, se aprecia que el $34,1 \%$ de los pacientes presentan lesiones laríngeas asociadas, siendo la más frecuente el sulcus vocalis, lo que se asemeja a lo publicado en otras series. Esto demuestra la importancia de un cuidadoso examen videolaringoestroboscópico, ya que algunas de estas lesiones asociadas pueden ser subdiagnosticadas.

\section{BIBLIOGRAFIA}

1. Altman KW. Vocal Fold Masses. Otolaryngologic Clinics of North America 2007; 40: 1091-108.

2. JoHns MM. Update on the etiology, diagnosis, and treatment of vocal fold nodules, polyps, and cysts. Current Opinion in Otolaryngology \& Head and Neck Surgery 2003; 11: 456-61.

3. Bouchayer M, CoRnut G. Microsurgery for benign lesions of the vocal folds. Ear, Nose and Throat Journal 1988; 67(6): 446-9, 452-4, 456-64.

4. Martins RH, Santana MF, Tavares El. Vocal Cysts: Clinical, Endoscopic, and Surgical Aspects. J Voice 2011; 25(1): 107-10.
El tratamiento de los quistes de cuerda vocal es quirúrgico. En nuestro estudio a todos los pacientes con diagnóstico de quiste de cuerda vocal se les indicó cirugía, pero por diversos motivos ajenos a la indicación médica, sólo el 34\% de ellos fueron finalmente operados. A ellos se les realizó microfonocirugía con técnica de microflap. Desde el punto de vista vocal, los resultados de esta técnica fueron satisfactorios en todos los casos, lo que se asoció a mejoría en la onda mucosa al examen videolaringoestroboscópico.

\section{CONCLUSIÓN}

Los quistes de cuerda vocal deben estar dentro de los diagnósticos diferenciales en los pacientes con disfonía, tanto en niños como en adultos. Estas lesiones se asocian a abuso vocal y frecuentemente afectan a personas con uso profesional de la voz. La evaluación videolaringoestroboscópica es fundamental, ya que la alteración de la onda mucosa es una característica determinante en estas lesiones, y por otro lado permite el diagnóstico de lesiones laríngeas asociadas, frecuentes en los pacientes con quistes de cuerda vocal. El tratamiento con microfonocirugía entrega resultados satisfactorios desde el punto de vista anatómico y funcional.
5. Zhukhovitskaya A, Battaglia D, Khosla SM, Murry T, SuLICA L. Gender and age in benign vocal fold lesions. Laryngoscope 2015; 125(1): 191-6.

6. Hogikyan ND, Sethuraman G. Validation of an instrument to measure voice-related quality of life (V-RQOL). J Voice 1999; 13(4): 557-69.

7. Rosen CA, Lee AS, Osborne J, Zullo T, Murry $T$. Development and validation of the voice handicap index-10. Laryngoscope 2004; 114(9): 1549-56.

8. Melo ECM, Brito LL, Brasil oco, Behlau M, Melo DM. Incidencia das lesoes laríngeas nao neoplásicas em pacientes com queixas vocais. Rev Bras Otorrinolaringol 2001; 67: 788-94.

Dirección: Carla Napolitano V.

Departamento de Otorrinolaringología de la Pontificia Universidad Católica de Chile

E mail: cnapolitanov@gmail.com 